\title{
Helicobacter pylori association with amoeba: a natural reservoir of the bacteria?
}

\author{
A. P. Alves de Matos****, F. F. Vale*** and J. M. B. Vitor**** \\ * Centro de Estudos do ambiente e do Mar (CESAM), Faculdade de Ciências da Universidade de \\ Lisboa, Campo Grande, 1749-016, Lisboa \\ ** Centro Hospitalar de Lisboa Central - HCC , Anatomia Patológica, R. da Beneficência 8, 1069-166 \\ Lisboa, Portugal \\ *** Faculdade de Engenharia, Universidade Católica Portuguesa, 2635-631 Rio de Mouro \\ **** iMed.UL, Faculdade de Farmácia da Universidade de Lisboa, 1649-003 Lisboa
}

Helicobacter pylori is a human pathogen involved in gastritis and gastric cancer whose mode of transmission remains unknown [1]. Association of $H$. pylori with humans is thought to date from remote antiquity and the bacterium has apparently evolved together with the human host [2]. A few studies have shown the presence of $H$. pylori in aquatic environments [3], which might provide a route of transmission of the bacteria to humans. A recent study has also disclosed the association of the bacteria with Acantamoeba castellanii [4]. Amoeba are known to harbor and promote the persistence of several human pathogens in the environment, representing a significant source of contamination in community and hospital acquired infections [5].

We have fed a strain of Acanthamoeba sp., isolated from dust of a Lisbon hospital, with a strain of $H$. pylori, and studied the infected amoeba by transmission electron microscopy. Pellets of the amoeba were fixed sequentially in glutaraldehyde, osmium tetroxide and uranyl acetate, dehydrated and embedded in Epon-Araldite mixture. Controls were done be feeding the amoebae with other bacteria including Escherichia coli, Proteus spp. and Shigella spp. Our results show that the amoeba were unable to grow with the $H$. pylori bacteria as nutrient, but grew and spread readily over the cultures of the other control bacteria. By transmission electron microscopy, the amoebae fed with H. pylori were seen to be filled with the bacteria located inside large vacuoles, and with little evidence of degradation.

Our results suggest that $H$. pylori may be among the growing group of amoeba-resistant microorganisms and that amoeba may play an important role in the maintenance of the bacteria in the environment. Further studies are being carried out to characterize the amoeba strain and the nature of the association of the amoeba with $H$. pylori.

\section{References}

1. Cave D.R., Gastroenterology 113: S9-14, 1997

2. Vale F.F. et al., BMC Microbiology 9: 193, 2009

3. Hulten K. et al., J Appl Microbiol 85: 282-286, 1998

4. Winiecka-Krusnell J. et al., Scand J Infect Dis 34: 253-256, 2002

5. Greub G. and Raoult D., Clin. Microbiol. Rev. 17: 413-433, 2004 


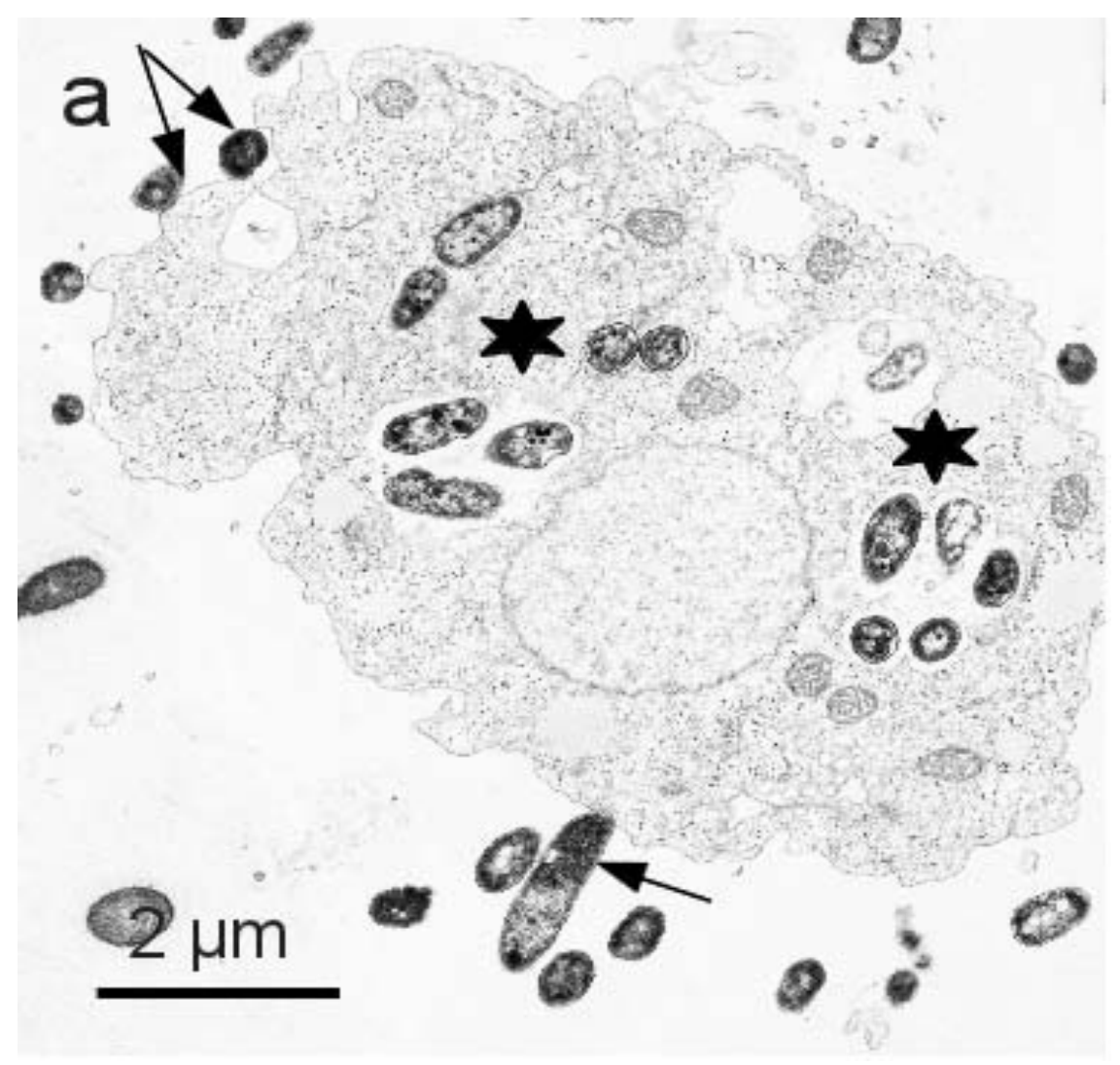

Figure 1. Acanthamoeba sp. culture (isolated from dust in the Curry Cabral Hospital) fed in a culture of $H$. pylori. Notice adhesion of bacteria to the surface of the cells (arrows), and ingested cells $\left(^{*}\right)$.

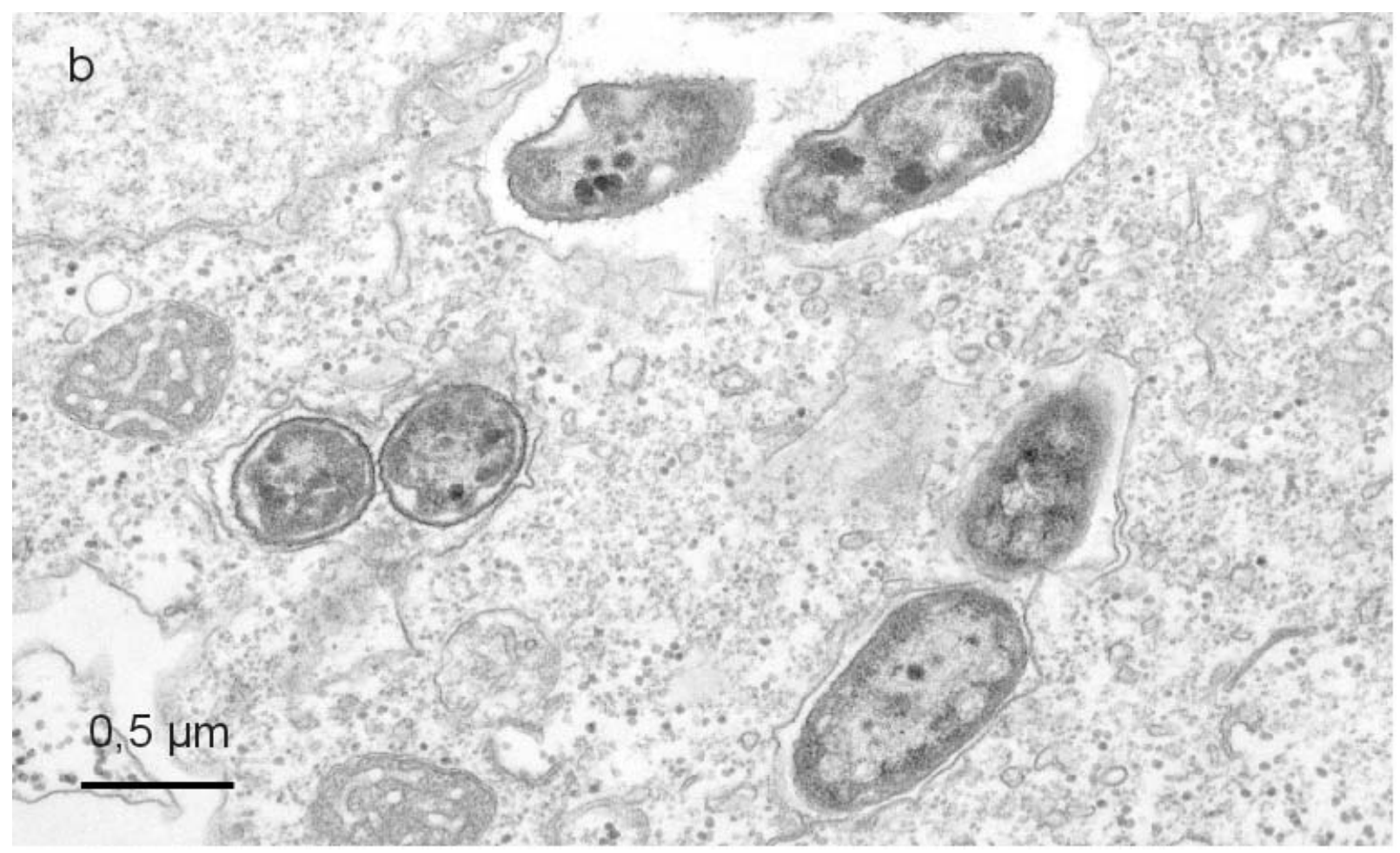

Figure 2. Acanthamoeba sp. culture (isolated from dust in the Curry Cabral Hospital) fed in a culture of H. pylori. Higher magnification of ingested bacteria inside phagocytic vacuoles. 